\title{
Recent Trends in Diabetes Treatment and Control in US Adults: A Geriatrician's Point of View
}

Fang M, Wang D, Coresh J, Selvin E. Trends in Diabetes Treatment and Control in U.S. Adults, 1999-2018. N Engl J Med. 2021;384(23):2219-2228. doi:10.1056/NEJMsa2032271

\section{Study Overview}

Objective. To update national trends in the treatment and risk factor control of diabetic patients from 1999 through 2018 in the US using data from the National Health and Nutrition Examination Survey (NHANES) with the goal of identifying population subgroups with the highest probability of having untreated risk factors.

Design. The authors conducted a cross-sectional analysis of data from NHANES focusing on adults with diabetes. They examined patient characteristics and medication use over time and estimated the prevalence of risk factor control and medication use. To minimize the effects of a small sample size, the survey years were pooled into 4-year intervals. The variables studied included glycated hemoglobin $\left(\mathrm{HbA}_{\mathrm{lc}}\right)$, blood pressure, serum cholesterol, medication use, sociodemographic characteristics, and weight status. For statistical analysis, logistic and multinomial logistic regression models were used to examine factors associated with treatment in participants who did not achieve targets for glycemic, blood pressure, and lipid control. Temporal trends were estimated using 2-piece linear spline models with 1 knot at inflection points.

Setting and participants. The NHANES program began in the early 1960s to monitor the health of the US population. In 1999, the survey became a continuous program combining interviews and physical examinations. The survey examines a nationally representative sample of about 5000 persons each year. This study included 6653 participants who were nonpregnant, aged older than 20 years, reported a diagnosis of diabetes from a physician, and participated in NHANES from 1999 through 2018.

Main outcome measures. The main outcome measures were temporal trends in risk factor control (glycemic, blood pressure, or lipid levels) and medication use (glucose lowering, blood pressure lowering, or lipid lowering medications), and number as well as class of drug used, from 1999 through 2018 in diabetic adults from the US participating in NHANES.

Results. Sociodemographic characteristics of the studied diabetes population-The age and racial or ethnic distribution of participants with diabetes were stable from 1999 through 2018, whereas participants with a college degree, higher income, health insurance, obesity, or long-standing diabetes increased during the same period. Trends in diabetes risk factor control-The trends for glycemic, blood pressure, and lipid control were nonlinear, with an inflection point around 2010. Glycemic

\section{Outcomes Research in Review SECTION EDITORS}

KatRina F. MATEO, PHD, MPH

CUNY School of Public Health New York, NY
DANIEL ISAAC, DO, MS

Michigan State University East Lansing, Ml
FRED Ko, MD, MS

Icahn School of Medicine at Mount Sinai New York, NY
TAISHI HIRAI, MD

University of Missouri

Columbia, $\mathrm{MO}$
WiLliam HUNG, MD, MPH

Icahn School of Medicine at Mount Sinai

New York, NY 


\section{Outcomes Research in Review}

control was defined as $\mathrm{HbA}_{1 \mathrm{c}}$ less than $7 \%$, blood pressure was considered controlled if less than $140 / 90 \mathrm{mmHg}$, and lipid was controlled if non-HDL cholesterol level was less than $130 \mathrm{mg} / \mathrm{dL}$. Although these chosen targets were based on the most recent clinical guidelines, the authors declared that they observed similar trends when alternative targets were used. The level of risk factor control improved in all diabetic patients from 1999 through 2010. However, the percentage of adult diabetic participants for whom glycemic control was achieved declined from $57.4 \%$ (95\% Cl, 52.9-61.8) in 2007-2010 to 50.5\% (95\% Cl, 45.8-55.3) in 2015-2018. Blood pressure control was achieved in $74.2 \%$ of participants (95\% Cl, 70.7-77.4) in $2011-2014$ but declined to $70.4 \%$ (95\% Cl, 66.7-73.8) in 2015-2018. Control in lipid levels improved during the entire study period; however, the rate of improvement heavily declined after 2007 with lipid target levels attained in $52.3 \%$ of participants $(95 \% \mathrm{Cl}, 49.2-55.3)$ in $2007-2014$ and $55.7 \%$ (95\% Cl, 50.8-60.5) in 2015-2018. Finally, the percentage of participants in whom targets for all 3 risk factors were simultaneously achieved plateaued after 2010 and was $22.2 \%(95 \% \mathrm{Cl}, 17.9-27.3)$ in 2015-2018. Trends in diabetes treatment-The use of glucose lowering drugs increased from $74.1 \%$ in $1999-2002$ to $82.7 \%$ in 2007-2010 and then stabilized. A shift toward a safer glucose lowering treatment choice was observed with a decline in the use of older glucose lowering medications such as sulfonylureas, which increases the risk of hypoglycemia, and an increase in the use of metformin, insulin, and newer agents such as sodium-glucose cotransporter 2 inhibitors.

Similarly, blood pressure lowering medication use rose from 1999-2002 to 2007-2010 and then stabilized, with increased use of first-line recommended treatments including angiotensin-converting enzyme inhibitors or angiotensin-receptor blockers. Likewise, statin use rose from $28.4 \%$ in $1999-2002$ to $56 \%$ in $2011-2014$ and then stabilized. The total number of drugs used culminated in 2011-2014 with 60\% of participants using more than 5 drugs and then leveled off to $57.2 \%$ in 2015-2018. Lastly, health insurance status and race or ethnicity impacted the likelihood of receiving monotherapy or combination drug therapy when targets for glycemic, blood pressure, or lipid control were not achieved.
Conclusion. Despite great progress in the control of diabetes and its associated risk factors between 1999 and 2010, this trend declined for glycemic and blood pressure control and leveled off for lipid control in adult NHANES participants with diabetes after 2010. Firstline treatments for diabetes and associated risk factors remain underused, and treatment intensification may not be sufficiently considered in patients with uncontrolled risk factors despite clinical guideline recommendations. The findings of this study may portend a possible population-level increase in diabetes-related illnesses in the years to come.

\section{Commentary}

The thorough understanding of trends in management of diseases is critical to inform public health policies and planning. Well designed clinical studies heavily influence the development of public health policies and clinical guidelines, which in turn drive real-world clinical practice. In a recent analysis utilizing data from NHANES, Fang et al $^{1}$ showed evidence of a general shift toward less intensive treatment of diabetes, hypertension, and hypercholesterolemia in adults living in the US during the last decade.

Similarly, in a separate study using NHANES data collected between 1999 and 2018 published in JAMA just 2 weeks after the current report, Wang et al ${ }^{2}$ confirms this declining trend in diabetes management with only $21.2 \%$ of diabetic adults simultaneously attaining glycemic, blood pressure, and lipid level targets during the same period. What led to the decline in more stringent risk factor and diabetes management since 2010 observed in these studies? One possible explanation, as suggested by Fang et al, is that major clinical trials from the late 2000s-including Action to Control Cardiovascular Risk in Diabetes, UK Prospective Diabetes Study, Action in Diabetes and Vascular Disease: Preterax and Diamicron Modified Release Controlled Evaluation, and Veterans Affairs Diabetes Trial-that assessed the effects of intensive glycemic control (with target $\mathrm{HbA}_{1 \mathrm{c}}<6.5 \%$ ) found that intensive treatment of diabetes compared to standard care had no cardiovascular benefit albeit increasing the risk of hypoglycemia. Thus, these trial findings may have translated into suboptimal diabetes treatment observed 


\section{Outcomes Research in Review}

in some NHANES participants. Wang et al propose that effective tailored approaches are needed to improve risk factor control in diabetic patients, such as enhance and maintain adherence to medications and healthy lifestyle behaviors, as well as better access to health care and therapeutic education.

The changes in recent trends in diabetes management have immense clinical implications. The authors of this study suggest a link between the recent relaxation of glycemic targets, as well as risk factor control, and a resurgence of diabetic complications such as lower limb amputation or stroke. Indeed, several recent studies indicate an upward trend or plateau in diabetic complications which had been decreasing in prevalence prior to 2010. ${ }^{3}$ For example, lower extremity amputation has surged by more than 25\% between 2010 and 2015, especially in young and middle-aged adults. ${ }^{4}$ Among the arguments brought forward that this recent resurgence in amputations is directly linked to worsening glycemic control is the fact that between 2007 and 2010, when glucose levels were best controlled within the previous 30-year period, amputations were also at the lowest levels. Moreover, data from the Centers for Disease Control and Prevention also show a 55\% increase in mortality (from 15.7 to 24.2 per 1000) among diabetic patients between 2010 and 2015. ${ }^{14}$ On the other hand, a growing number of studies show that an increase of inappropriate treatment intensification-reaching $\mathrm{HbA}_{\mathrm{cc}}$ levels that are way below the recommended targets-is associated with adverse consequences in diabetic patients particularly in those aged more than 65 years..$^{5-7}$ These seemingly contradictory findings highlight the importance of a personalized and thoughtful approach to the management of diabetes and its risk factors. As an example, an increase in the use of newer and safer glucose lowering drugs (eg, sodium-glucose cotransporter 2 inhibitors, glucagon-like peptide 1 receptor agonists, and dipeptidyl peptidase 4 inhibitors) can help achieve better $\mathrm{HbA}_{1 \mathrm{c}}$ goals with a reduced risk of hypoglycemic episodes as recently shown by a Danish study. ${ }^{8}$ In this study, the authors concluded that the reduction of the rate of hypoglycemic episodes leading to hospitalization in Denmark was directly linked to the use of these safer and newer glucose lowering drugs.
A discussion on the specifics of trends in diabetes treatment and control must include considerations in older adults aged more than 65 years who constitute more than $40 \%$ of the diabetic population. Despite the high prevalence of diabetes in this vulnerable population, such data are still insufficient in the literature and are critically needed to inform public health policies and clinical guidelines. In epidemiological studies focusing on diabetic complications from the last 10 years, concerning increases have been observed in younger ${ }^{9}$ and middle-aged adults while remaining stable in older adults. However, the risk of hypoglycemia or severe hypoglycemia remains high in older adults living in nursing facilities, even in those with an elevated $\mathrm{HbA}_{1 \mathrm{c}}$ of greater than 8\%.7 Moreover, in light of more relaxed $\mathrm{HbA}_{\mathrm{lc}}$ treatment goals for older frail adults as recommended by international guidelines since 2010,10,11 recent findings from the French GERODIAB cohort show an increased mortality (hazard ratio, 1.76) in type 2 diabetics aged 70 years and older with $\mathrm{HbA}_{1 \mathrm{c}}$ greater than or equal to $8.6 \% .^{12}$ Similarly, a 5 -year retrospective British study from 2018 which included patients aged 70 years and older, shows an increased overall mortality in those with $\mathrm{HbA}_{\mathrm{lc}}$ greater than $8.5 \% .^{13}$ Taken together, further age-stratified analysis utilizing data from large cohort studies including NHANES may help to clarify national trends in diabetes treatment and risk factor control as well as diabetic complications specific to the geriatric population. By being better informed of such trends, clinicians could then develop treatment strategies that minimize complications (eg, hypoglycemia, falls) while achieving favorable outcomes (eg, reduce hyperglycemic emergencies, improve survival) in frail older patients.

\section{Applications for Clinical Practice}

The understanding of population-wide trends in diabetes control is critical to planning public health approaches for the prevention and treatment of this disease and its complications. In older adults, the high risk of hypoglycemic events and insufficient epidemiological data on trends of diabetes control hinder diabetes management. Personalized treatment targets taking into account geriatric syndromes and general health status, as well as 
multidisciplinary management involving endocrinologists, geriatricians, and clinical pharmacists, are necessary to optimize care in older adults with diabetes.

\section{-Rachel Litke, MD, PhD, and Fred Ko, MD, Icahn School of Medicine at Mount Sinai, New York City, New York \\ doi:10.12788/jcom.0060}

\section{References}

1. Fang M, Wang D, Coresh J, Selvin E. Trends in Diabetes Treatment and Control in U.S. Adults, 1999-2018. N Engl J Med. 2021;384(23):2219-28. doi:10.1056/NEJMsa2032271

2. Wang L, Li X, Wang Z, et al. Trends in Prevalence of Diabetes and Control of Risk Factors in Diabetes Among US Adults, 1999-2018. JAMA. 2021. doi:10.1001/jama.2021.9883

3. Gregg EW, Hora I, Benoit SR. Resurgence in Diabetes-Related Complications. JAMA. 2019;321(19):1867-8. doi:10.1001/ jama.2019.3471

4. Caruso P, Scappaticcio L, Maiorino Ml, et al. Up and down waves of glycemic control and lower-extremity amputation in diabetes. Cardiovasc Diabetol. 2021;20(1):135. doi:10.1186/ s12933-021-01325-3

5. Bongaerts B, Arnold SV, Charbonnel BH, et al. Inappropriate intensification of glucose-lowering treatment in older patients with type 2 diabetes: the global DISCOVER study. BMJ Open Diabetes Res Care. 2021;9(1)e001585. doi:10.1136/ bmjdrc-2020-001585
6. Lipska KJ, Ross JS, Wang Y, et al. National trends in US hospital admissions for hyperglycemia and hypoglycemia among Medicare beneficiaries, 1999 to 2011. JAMA Intern Med. 2014;174(7):11161124. doi: 10.1001/jamainternmed.2014.1824

7. Bouillet B, Tscherter P, Vaillard L, et al. Frequent and severe hypoglycaemia detected with continuous glucose monitoring in older institutionalised patients with diabetes. Age Ageing. 2021;afab128. doi: 10.1093/ageing/afab128

8. Jensen MH, Hejlesen O, Vestergaard P. Epidemiology of hypoglycaemic episodes leading to hospitalisations in Denmark in 19982018. Diabetologia. 2021. doi: 10.1007/s00125-021-05507-2

9. TODAY Study Group, Bjornstad P, Drews KL, et al. Long-Term Complications in Youth-Onset Type 2 Diabetes. N Engl J Med. 2021;385(5):416-426. doi: 10.1056/NEJMoa2100165

10. Sinclair AJ, Paolisso G, Castro M, et al. European Diabetes Working Party for Older People 2011 clinical guidelines for type 2 diabetes mellitus. Executive summary. Diabetes Metab. 2011;37 Suppl 3:S27-S38. doi:10.1016/S1262-3636(11)70962-4

11. Kirkman MS, Briscoe VJ, Clark N, et al. Diabetes in older adults. Diabetes Care. 2012;35(12):2650-2664. doi: 10.2337/dc12-1801

12. Doucet J, Verny C, Balkau B, et al. Haemoglobin A1C and 5-year all-cause mortality in French type 2 diabetic patients aged 70 years and older: The GERODIAB observational cohort. Diabetes Metab. 2018;44(6):465-472. doi: 10.1016/j.diabet.2018.05.003

13. Forbes A, Murrells T, Mulnier H, Sinclair AJ. Mean HbA1c, HbA1c variability, and mortality in people with diabetes aged 70 years and older: a retrospective cohort study. Lancet Diabetes Endocrinol. 2018;6(6):476-486. doi: 10.1016/S2213-8587(18)30048-2

14. US Centers for Disease Control and Prevention. US diabetes surveillance system and diabetes atlas, 2019. https://www.cdc.gov/ diabetes/data 